\title{
Papillon Lefevre syndrome: Bridge between Dermatologist and Dentist
}

\author{
Anshu Singla ${ }^{1}$, Soheyl Sheikh ${ }^{2}$, Sanjeev Kumar Jindal ${ }^{3}$, Rajdeep Brar ${ }^{4}$
}

${ }^{1}$ M.D.S., Senior Lecturer, Department of Pedodontics \& Preventive Dentistry, Bhojia Dental College \& Hospital, Vill. Budh, Baddi.

${ }^{2}$ M.D.S., Professor, Department of Oral Medicine and Radiology, M.M. College of Dental Sciences \& Research, Mullana, Ambala, Haryana, India.

${ }^{3}$ M.D.S., Senior Lecturer, Department of Oral Medicine and Radiology, Bhojia Dental College \& Hospital, Vill. Budh, Baddi.

${ }^{4}$ M.D.S., Senior Lecturer, Department of Oral Medicine and Radiology, Swami Devi Dayal Hospital \& Dental College, Barwala, Ambala

Correspondence:

Dr. Anshu Singla,

Department of Pedodontics \& Preventive Dentistry,

Bhojia Dental College \& Hospital, Vill. Budh,

Baddi, Tehsil Nalagarh,

District Solan, Himachal Pradesh, India

E-mail;saj2908@yahoo.com

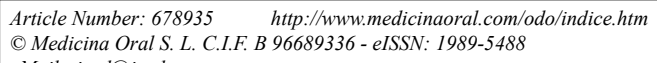

\begin{abstract}
Papillon-Lefevre syndrome is a rare autosomal recessively inherited condition which shows features common to both dentistry and dermatology. This disease is characterized by palmoplantar hyperkeratosis and severe periodontitis. Therefore both the dentist and the dermatologist should be aware of the same because an early diagnosis of the syndrome can help to preserve the teeth by early institution of treatment, using a multidisciplinary approach. Here we present a case report of Papillon-Lefevre syndrome, along with a comprehensive review of the etiology, pathology, clinical features, differential diagnosis and management of the condition.
\end{abstract}

Key words: Papillon Lefevre syndrome, palmoplantar hyperkeratosis, periodontosis. 


\section{Introduction}

Palmoplantar keratoderma refers to a heterogeneous group of disorders characterized by thickening of the palms and soles. They can be hereditary, acquired, or associated with syndromes.

In 1924, two French physicians Papillon and Lefevre described a brother and sister with a condition characterized by palmoplantar hyperkeratosis associated with severe, early onset periodontitis and premature loss of primary and permanent teeth (1-3). Common cutaneous changes include well-demarcated erythematous hyperkeratotic lesions on the palms, soles, dorsum of the hands, and the interphalangeal joints. These plaques may occur focally, but usually involve the entire surface of the palms and soles, sometimes extending onto the dorsal surfaces of the hands and feet (1). The inheritance is autosomal recessive and consanguinity is a notable feature in many patients. Other features of the syndrome which have been reported less frequently, include psoriasiform plaques of the elbows and knees, nail changes, calcification of the dura, and recurrent pyogenic skin infections (1-6).

This disease usually has its onset between the ages of 1 to 4 years. Males and females are equally affected. There is no racial predominance. Its prevalence is estimated to be 1 to 4 per million in the general population with a carrier rate of 2 to 4 per 1000 (4).

Here we report a case of Papillon-Lefevre syndrome in a 16 year old boy. He was the only member affected in his family. In his case a change of weather had an effect on palmoplantar hyperkeratosis.

\section{Case Report}

A 16 year-old male patient presented to the Department of Oral Medicine and Radiology, complaining of teeth mobility, esthetic problems and difficulty in eating since 1 year due to loss of permanent teeth. Past dental history revealed loss of permanent teeth 1 year back due to excessive mobility and also early shedding of deciduous dentition. He also gave a history of thickening and scaling of the skin of the palms and soles since childhood which aggravates during the monsoon season. Past medical history revealed frequent upper respiratory infections. The rest of the family members including parents and two siblings were apparently normal.

\section{Clinical examination}

On physical examination, the patient was noted to have well-demarcated, yellow keratotic plaques of his palms (Fig. 1) and soles (Fig. 2) bilaterally with a spillover onto the dorsal surface of the hands and feet. The knees and elbows were also affected, but to a less severe degree than the palmoplantar surfaces.

Intraoral examination revealed missing $12,14,21,22$, $26,31,42,46$. There was pathological migration of the remaining teeth (Fig. 3). The gingiva around the teeth

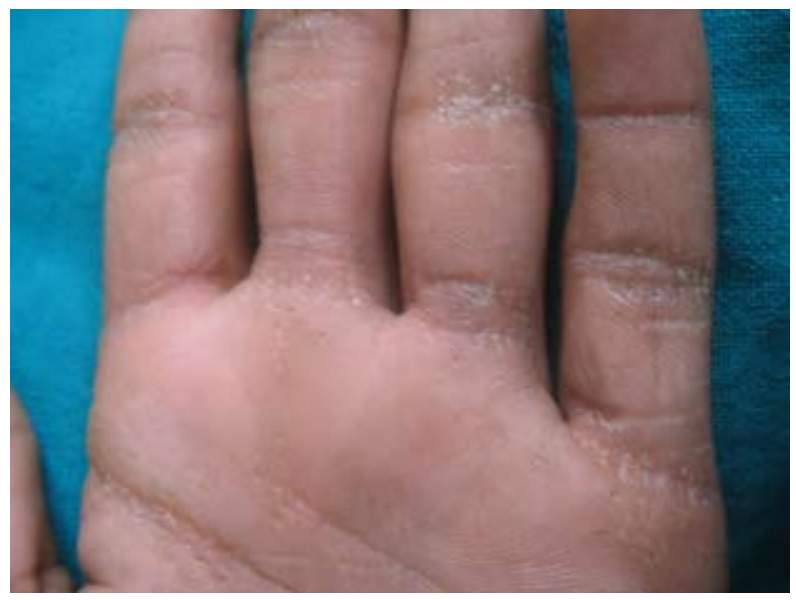

Fig. 1. Showing hyperkeratosis of palmar surface

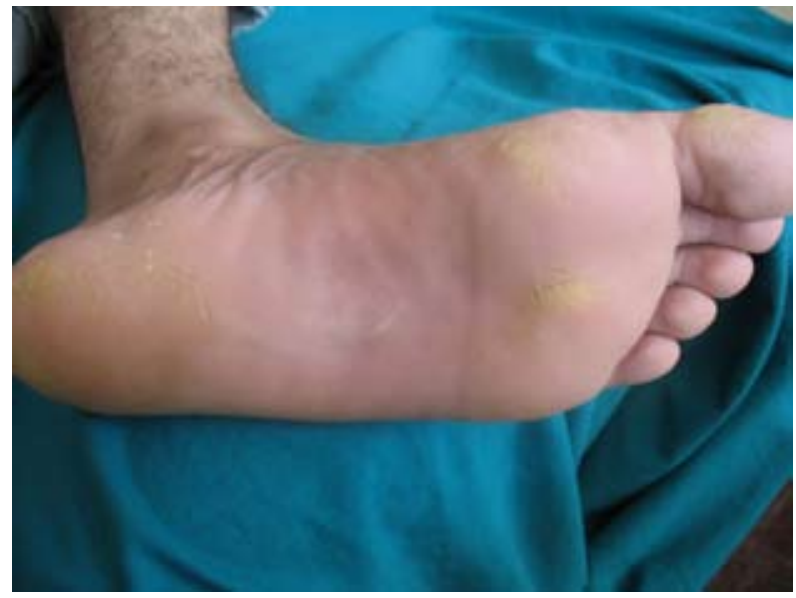

Fig. 2. Showing hyperkeratosis of plantar surface

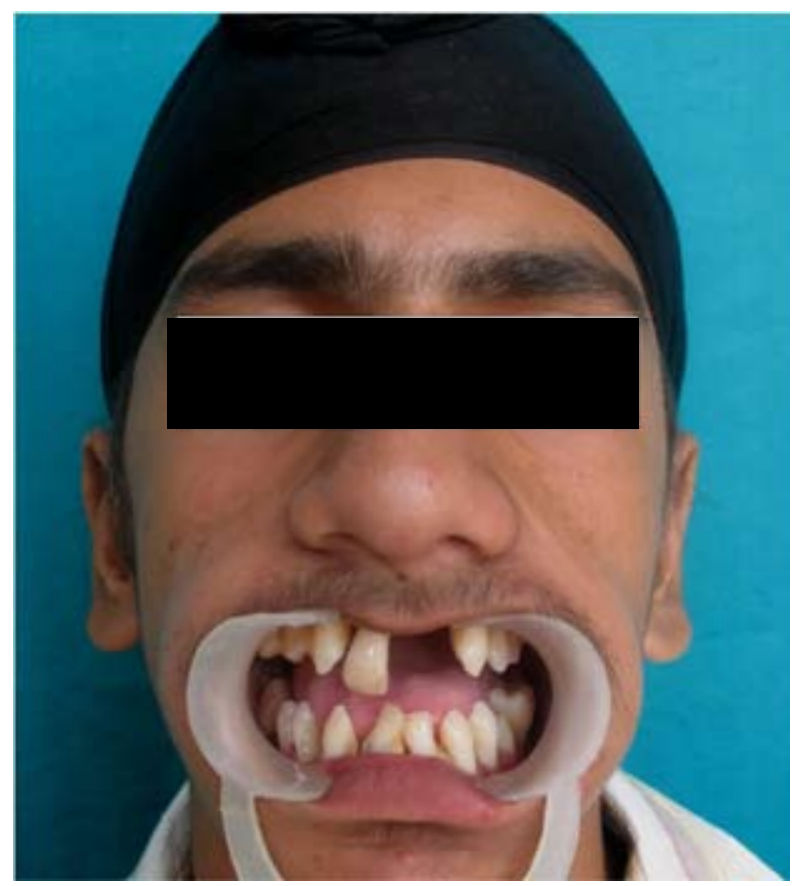

Fig. 3. Showing severe periodontitis. 
was inflamed, swollen and tender while the oral mucosa covering the edentulous area appeared normal. Generalized plaque accumulation along with halitosis was present. The teeth were mobile with pathological periodontal pockets from which pus exuded upon probing. Based on the history and clinical findings, a provisional diagnosis of Papillon-Lefevre syndrome was made.

A panoramic radiograph was obtained which revealed generalized horizontal alveolar bone loss. Besides 35 and 38 were vertically impacted. Routine laboratory investigations were within normal limits. Ocular examination revealed no abnormality. A microbiologic examination by polymerase chain reaction analysis identified the presence of Actinomyces actinomycetemcomitans, Porphyromonas gingivalis, Prevotella intermedia and Treponema denticola.

Treatment

Extraction of grade III mobile teeth namely 11, 25, 32 and 41 was done. The patient was put on Metronidazole tablets $400 \mathrm{mg}$ twice daily and chlorhexidine mouth washes to be used 2-3 times daily. This was followed by oral prophylaxis and complete oral rehabilitation. Consultation of a dermatologist was taken for evaluation of hyperkeratosis of palmar and plantar surfaces.

\section{Discussion}

Papillon-Lefevre syndrome is a rare disorder that is inherited in an autosomal recessive manner, that is, both parents are phenotypically healthy and there is no family history of the disease, other than the affected person and possibly some siblings. Both parents must carry the autosomal gene for the syndrome to appear in their offspring. When 2 such carriers mate, there is a $25 \%$ chance of producing affected offspring. Our case falls in this category (1-2).

The etiology of Papillon-Lefevre syndrome is still unknown. Three main factors have been suggested as being responsible for the initiation and progression of Papillon-Lefevre syndrome.

Firstly, an impairment of neutrophil chemotaxis, phagocytosis and bactericidal activities accompanied by a decrease in cell migration was noted (3,7-10). Secondly, the presence of virulent gram-negative anaerobic pathogens (Actinobacillus actnomycetemocomitans) in the periodontal plaques and in periodontal pockets was noted. It was inferred that they might act as trigger factors $(3-4,11)$. Thirdly, a defect of immune-mediated mechanisms including reduced lymphocyte response to pathogens, depression of helper/suppressor T cells ratio, deficient monocytic function, elevation of serum IgG and degenerative changes of plasma cells were identified $(2-3,6)$.

Recently, genetic examinations point to the PapillonLefevre syndrome gene locus in these patients. The gene responsible for it has been localized to chromoso- me 11q14-21 where the cathepsin $C$ gene is, encoding a lysosomal protease, in the interval between D11S4082 and D11S931. Subsequently, inactivating mutations were identified in this gene and an almost total loss of cathepsin $\mathrm{C}$ activity was shown in patients with Papillon-Lefevre syndrome (4-6). An interesting feature of the cathepsin $\mathrm{C}$ gene is that mutations in this gene also result in two other closely related conditions: the Haim-Munk syndrome, and prepubertal periodontitis. A common clinical manifestation in all three syndromes is severe early-onset periodontitis.

Seasonal variations of skin lesions in Papillon-Lefevre syndrome are not well documented but in our case the palmoplantar hyperkeratosis was aggravated during the monsoon season. This is an unusual finding.

When there is premature loss of deciduous and/or permanent teeth, one should also consider Acrodynia which is also known as Feer's syndrome. This condition is usually caused by mercury intoxication. Here one may observe a red desquamative process involving both the extremities. But in addition there are erythocyanosis, muscle pain, insomnia, sweating, tachycardia and psychic disturbances. The teeth erupt prematurely, have dystrophic enamel, and are shed prematurely. Acrodynia is seen in children between the ages of six months and four years (1).

Another condition of interest in differential diagnosis is Hypophosphatasia. In addition to the clinical features of knock-knee, bowing of femur and tibia and enlarged wrists. The teeth are prematurely shed and are hypoplastic. Diagnosis can be made on basis of increased amounts of phosphoethanolamine in the urine (1).

Another condition characterized by progressive gangrenous lesions involving the gingiva and alveolar bone, resulting in exfoliation of the teeth is Acatalasemia or Takahara's syndrome. This is also transmitted as an autosomal recessive trait but has rarely been observed outside Japan (1).

In cyclic neutropenia severe periodontal disease may also be present but palmoplantar hyperkeratosis is absent $(1,2)$.

Other condition to be considered in differential diagnosis of Papillon-Lefevre syndrome are palmoplantar hyperkeratosis of Unna Thost, mal de Meleda, Howel-Evans syndrome, keratosis punctata, keratoderma hereditarium mutilans (Vohwinkel's syndrome), and Greither's syndrome. However while these entities are associated with palmoplantar hyperkeratosis but there is no periodontopathy (2).

Incidence of this rare entity is increasing in recent times and since it involves severe irreparable destruction of dentition at an early age, so the dental professional has an important role to play and hence should be well thorough with etiological factors and effective treatment of Papillon-Lefevre syndrome. 
Oral retinoids, such as acitretin, etretinate, and isotretinoin, have been reported to be beneficial in treating both the cutaneous and dental defects in Papillon-Lefevre syndrome. An important point to note is that if retinoid therapy is started during the eruption of the permanent teeth, it can result in the development of normal dentition $(2,4-6,12,13)$.

Thus both the dermatologist and dental professional can help to save the permanent dentition if they diagnose this disease during childhood.

\section{References}

1. Ashri NY. Early diagnosis and treatment options for the periodontal problems in Papillon-Lefèvre syndrome: a literature review. J Int Acad Periodontol. 2008;10:81-6.

2. Hattab FN, Amin WM. Papillon-Lefèvre syndrome with albinism: a review of the literature and report of 2 brothers. Oral Surg Oral Med Oral Pathol Oral Radiol Endod. 2005;100:709-16.

4. Angel TA, Hsu S, Kornbleuth SI, Kornbleuth J, Kramer EM. Papillon-Lefevre syndrome: a case report of four affected siblings. J Am Acad Dermatol. 2002;46:S8-10.

5. Allende LM, Moreno A, de Unamuno P. A genetic study of cathepsin $\mathrm{C}$ gene in two families with Papillon-Lefèvre syndrome. Mol Genet Metab. 2003;79:146-8.

6. Pilger U, Hennies HC, Truschnegg A, Aberer E. Late-onset Papillon-Lefèvre syndrome without alteration of the cathepsin $\mathrm{C}$ gene. J Am Acad Dermatol. 2003;49:S240-3.

7. Janjua SA, Khachemoune A. Papillon-Lefèvre syndrome: case report and review of the literature. Dermatol Online J. 2004;10:13.

8. Ghaffer KA, Zahran FM, Fahmy HM, Brown RS. Papillon-Lefèvre syndrome: neutrophil function in 15 cases fron 4 families in Egypt. Oral Surg Oral Med Oral Pathol Oral Radiol Endod. 1999;88:320-5.

9. Liu R, Cao C, Meng H, Tang Z. Leukocyte functions in 2 cases of Papillon-Lefèvre syndrome. J Clin Periodontol. 2000;27:69-73.

10. Lundgren T, Parhar RS, Renvert S, Tatakis DN. Impaired cytotoxicity in Papillon-Lefèvre syndrome. J Dent Res. 2005;84:414-7.

11. González JR, Chabrier L, Rodriguez RJ. Papillon-Lefevre syndrome: a case report and review of the literature. P R Health Sci J. 1997; 16:279-81.

12. Lundgren T, Crossner CG, Twetman S, Ullbro C. Systemic retinoid medication and periodontal health in patients with Papillon-Lefèvre syndrome. J Clin Periodontol. 1996;23:176-9.

13. el Darouti MA, Al Raubaie SM, Eiada MA. Papillon-Lefèvre syndrome. Successful treatment with oral retinoids in three patients. Int J Dermatol. 1988;27:63-6. 